\section{Presencia de alergias en menores por consumo temprano de alimentos en Barranquilla, Colombia}

\author{
Allergies in children due to early food consumption \\ in Barranquilla, Colombia
}

\author{
Karol Cervantes-De La Torre, Francisco Guillen-Grima, \\ Inés Aguinaga-Ontoso y Adel Mendoza-Mendoza
}

Recibido 28 febrero 2017 / Enviado para modificación 14 septiembre 2017 / Aceptado 16 enero 2018

\section{RESUMEN}

Objetivo Determinar la prevalencia de síntomas asociados a asma, rinitis y eccema relacionada con la ingesta de alimentos en menores de seis a siete años.

Metodología Estudio descriptivo de prevalencia de asma, rinitis y eccema y la relación con el consumo de alimentos. Información obtenida en 1520 escolares del Distrito de Barranquilla y su área metropolitana. Se empleó metodología ISSAC procesándose la información mediante el software SPSS versión 24 El cuestionario fue contestado por el acudiente del menor y solo se tuvieron en cuenta los que tenían debidamente diligenciado los consentimientos informados Se hizo análisis empleando Chi-cuadrado, y el valor $\mathrm{P}$ de significancia con un $95 \%$ de confianza.

Resultados Para los 1520 menores encuestados el alimento que presentó relación con alergias fue el pescado asociándolo con el asma, evidenciando que en la población puede ser factor protector para la aparición de eccema mostrando estadísticamente una $\mathrm{P}<0,05$, un Chi-cuadrado: 4,566, IC 95\% [0,3;0,8].

Conclusiones De los alimentos analizados el pescado es uno que presentó relación y se asoció como factor protector para el eccema, el consumo de la leche de vaca se relacionó como factor protector para rinitis; en lo referente a los demás alimentos y las alergias el estudio no es conclusivo en cuanto a si la ingesta temprana es un factor predisponente o desencadenante de ellas en los menores, o no lo son.

Palabras Claves: Hipersensibilidad a los alimentos; dieta; niño; región del caribe (fuente: DeCS, BIREME).

\section{ABSTRACT}

Objective To determine the prevalence of symptoms associated with asthma, rhinitis and eczema with food intake in children under six years of age.

Materials and Methods Descriptive study on the prevalence of asthma, rhinitis and eczema and their correlation with food consumption. Information was obtained from 1 520 schoolchildren of the District of Barranquilla and its metropolitan area. The ISSAC method was used to process the information, which was provided by the child's guardian, using the software SPSS version 24 . Only duly completed questionnaires with informed consents were considered. Analysis was made using a chi-squared test, with a significant $P$ value and $95 \%$ confidence.

Results In the 1520 children surveyed, the type of food related to allergies was fish, associated with asthma, showing that this may be a protective factor for the population regarding the onset of eczema, with a statistically a $P$ value of $<0.05$, chi-square of 4.566 , and $\mathrm{Cl}$ of $95 \%$ [0.3;0.8].

Conclusions The analyzed foods showed that fish is related and associated as a protective factor for eczema, while the consumption of cow's milk was related as a protec-
KC: Fisioterapeuta. Ph. D. Salud Pública. Facultad de Ciencias de la Salud, Universidad Libre Barranquilla. Barranquilla, Colombia.

kcervantes@unilibrebaq.edu.co.

FG: Licenciado en Medicina y Cirugía. Ph. D. Medicina. Departamento de Ciencias de la Salud. Universidad Pública de Navarra. Pamplona, España.f.guillen.grima@unavarra.es

IA: Licenciada en Medicina y Cirugía. Ph. D. Medicina y Cirugía. Departamento de Ciencias de la Salud. Universidad Pública de Navarra. Pamplona. España.ines.aguinaga@unavarra.es AM: Ing. Químico. M. Sc. Ingeniería Industrial. Facultad de Ingeniería. Universidad del Atlántico. Barranquilla, Colombia. adelmendoza@uniatlántico.edu.co 
tive factor for rhinitis. Regarding other foods and allergies, the study is not conclusive as to whether their early intake is a predisposing factor or trigger in children.

Key Words: Food hypersensitivity; diet; child; caribbean region (source: $M e S H, N L M$ ).

$\mathrm{D}$ ebido a los rápidos ritmos en los que transcurre la vida actualmente se han producido cambios en los estilos de vida, entre estos cambios se puede resaltar la alimentación, también encontramos cambios en lo que respecta al manejo de recursos medioambientales entre esos el aumento de la deforestación, contaminación de fuentes hídricas y la contaminación del aire entre otros. Esto último ha desencadenado el incremento de enfermedades como las alergias, primando las que involucran a las vías aéreas; lo anterior se respalda en investigaciones que han demostrado la relación que puede llegar a un $20 \%$ entre la contaminación ambiental y estas enfermedades, siendo esta cifra la de países industrializados (1-2).

En la investigación en salud resulta relevante para su personal el estudio de las enfermedades alérgicas, y sus posibles causas ya que a la postre representan un elevado costo no solo en dinero sino también en incapacidades bien sea laborales o para la realización de otra actividad que corresponda a la vida diaria, dichas enfermedades provocan un descenso en la calidad de vida no solo en el que la padece también involucra en muchas ocasiones al cuidador ya que se ha reportado en la literatura episodios de estrés en ellos debido a la actividad que realizan.

Estas patologías son de especial cuidado ya que se evidencia su aumento (3-4), dentro de las enfermedades alérgicas, solo el asma ha presentado un incremento de un $30 \%$ en el último decenio (5). Actualmente se llega a estimar que la población mundial que padece alergias va de un $10 \%$ a $15 \%$ (6) y se prevé que para este siglo la mitad de la población padecerá algún tipo de alergia pasando así a ser una situación problema en salud pública de gran envergadura a la cual hay que apuntar en materia de prevención (7), en gran medida debido a que cuando se padece estas enfermedades siendo jóvenes tienden a convertirse en procesos crónicos cuando se pasa a la etapa de adultez aumentando aún más los costos derivados a su atención en salud (8).

Uno de los factores desencadenante de las alergias es la alimentación, se ha encontrado una relación que va del $6 \%$ al $8 \%$ entre ciertos alimentos y las alergias en menores de edad (9).En países como México las alergias están en los 20 primeros puestos en las enfermedades que se dan en menores de 5 a 14 años (10). Los resultados encontrados entre las prevalencias de las alergias y su posibles variables causantes son variados esto se puede deber a las diversas metodologías empleadas en la realización de los estudios, la geografía donde este se realizó, y los factores sociales de los individuos entre otros (11).

La saludable alimentación desde edades tempranas contribuye a un desarrollo óptimo del individuo no solo a nivel biológico sino también a nivel cognitivo, colaborando de este modo a la prevención de aparición de futuras enfermedades incluidas las alergias (12-13). Continuando con la alimentación, lo social, cultural y educativo influyen en la forma de cómo alimentarnos, incluso cómo nutrimos a nuestros descendientes, está demostrado que es él o la cuidadora primaria quien determina qué tipo de alimentos se deben ingerir en las primeras etapas del desarrollo, siendo este hecho de vital importancia para un crecimiento optimo del individuo (14) entre más temprano se consuman alimentos procesados aumenta el riesgo de desarrollar algún tipo de alergia asociada a ellos (15).

Existen diferentes metodologías empleadas en investigación para detectar asociaciones entre ciertos factores y las alergias. El International Study of Asthma and Allergies in Childhood (ISSAC) dentro de las variables que estudia evalúa a los alimentos que frecuentemente consumen los menores y busca encontrar asociación entre ellos y el asma, la rinitis y el eccema. Es importante precisar que el estudio Iss AC es un estudio multicentrico en donde ha habido reportes de 156 centros de investigación, para América Latina han reportado 18 centros lo cual ha permitido que sus resultados sean confiables (6). Proporcionando información sobre como es el comportamiento de las alergias en diversas zonas geográficas, tomando este estudio como referente se aplicó el cuestionario ISSAC a menores de seis a siete años escolarizados en Barranquilla con el objetivos de poder determinar si existe correlación entre enfermedades alérgicas y el consumo de alimentos como el pescado, huevo y leche de vaca a temprana edad.

\section{MÉTODOLOGÍA}

Se realizó un estudio transversal a 1520 niños y niñas con edades entre los seis y siete años debidamente matriculados en colegios de Barranquilla y algunos pertenecientes a su área metropolitana entre los años 2014 y 2015 con el propósito de determinar la correlación entre el consumo temprano de alimentos como: carne, huevo y leche de vaca y la manifestación de enfermedades alérgicas tales como asma, rinitis y eccema, se empleó para ello 
el cuestionario ISSAC donde se seleccionaron las preguntas relacionadas con la ingesta temprana de los alimentos antes mencionados. El cuestionario fue resuelto por los padres o acudientes del menor, según lo que manifestaran, solo se tuvieron en cuenta los que eran devueltos con el consentimiento informado diligenciado. El método de muestreo que se utilizo fue el muestreo por conglomerados, Para la selección de los colegios se tuvo en cuenta que estos debían estar en base de datos oficiales del Ministerio de Educación Nacional, también que debían estar registrados en la base de datos de la Secretaria de Educación del Distrito de Barranquilla

Para la selección de los individuos se tuvo en cuenta la variable edad, en donde los participantes debían reportar seis o siete años cumplidos al momento de participar en la investigación y también estar en la base de datos del plantel educativo.

En el análisis estadístico se aceptó un nivel de significancia por debajo del o.05. Complementándolo con el cálculo del Odds ratio y Chi-cuadrado.

\section{RESULTADOS}

Dentro de las características de la población tenemos que se aplicaron 1520 encuestas, resultando 710 niños $(46,71 \%)$ y 810 niñas $(53,29 \%)$; las edades comprendidas fueron de seis y siete años cumplidos, con edad promedio de 6,434 años. Entre los resultados a resaltar encontramos que para la relación entre el asma como enfermedad alérgica y el consumo temprano de huevo en los menores el número de casos validos fue de 1349 , de los cuales 240 reportan haber consumido el alimento antes de los seis meses y 1109 después de esa edad, no se encontró relación entre las variables hallándose un Chi-cuadrado de: 0,001 un valor $\mathrm{P}>0,05$ y un or: 1,o por lo anterior se rechaza la hipótesis nula, lo que refiere que el consumo de huevo a temprana edad no presenta relación con la manifestación del asma bronquial. En la rinitis y su relación con el consumo de huevo el total de casos validos fue de 1323 de los cuales 240 contestaron haber consumido antes de los seis meses y 1083 después de los seis meses se encontró una P>0,05 acompañado de un Chi-cuadrado de: 3,422 y una or de 0,79 concluyéndose con un 95\% de confianza que tampoco están relacionados. Para el eccema los casos validos fueron de 1 296, los que contestaron haber consumido antes de los 6 meses fueron 240 menores y después de los 6 meses 1056 los resultados arrojados fueron: un Chi-cuadrado de: 3,636; P>0,05 y OR: 1,2 no considerándose en el estudio la relación entre la ingesta temprana del alimento y la aparición de la alergia (Tabla 1).
Tabla 1. Presencia de asma, rinitis y eccema y consumo de huevo

\begin{tabular}{lccc}
\hline Alergia & $\begin{array}{c}\text { Chi-cuadrado de } \\
\text { Pearson }\end{array}$ & $\begin{array}{c}\text { Significación } \\
\text { asintótica (bilateral) }\end{array}$ & $\begin{array}{c}\text { Odds ratio } \\
\text { (OR) }\end{array}$ \\
\hline Asma & 0,001 & 0,976 & 1,00 \\
Rinitis & 3,422 & 0,064 & 0,79 \\
Eccema & 3,636 & 0,057 & 1,20 \\
\hline
\end{tabular}

En lo que corresponde al consumo de pescado y su referente al asma, el número de casos validos fueron de 1385 , siendo los que consumieron antes de los seis meses 250 y posterior a esa edad 1135 , se obtuvo en el análisis un Chi-cuadrado: 32,374; $\mathrm{P}<0,05$ y una OR: 2,40 lo que indica que hay una relación entre las variables es decir que con un $95 \%$ de confianza la ingesta de pescado a temprana edad si favorece la presencia de asma en los menores. En el caso de la rinitis el total de muestra valida ascendió a 1 359, los que consumieron el alimento antes de los seis meses fueron en total 250 y después de seis meses 1 109, los resultados obtenidos fueron: un OR: 1,06 y un Chi-cuadrado: 0,17; para una P>0,05, no evidenciando la relación entre el consumo del alimento y la aparición de la alergia. Para el eccema y su relación con el pescado los casos validos fueron de 1332 , siendo los que consumieron tempranamente 250 y después de los 6 meses 1 082, se obtuvo una $\mathrm{P}<0,05$, un Chi-cuadrado: 4,566 y un or: o,62, lo cual nos sugiere que el pescado puede actuar como protector para esta enfermedad (Tabla 2).

Tabla 2. Presencia de asma, rinitis y eccema y consumo de pescado

\begin{tabular}{lccc}
\hline Alergia & $\begin{array}{c}\text { Chi-cuadrado de } \\
\text { Pearson }\end{array}$ & $\begin{array}{c}\text { Significación } \\
\text { asintótica (bilateral) }\end{array}$ & Odds ratio (OR) \\
\hline Asma & 32,374 & 0,000 & 2,40 \\
Rinitis & 0,172 & 0,678 & 1,06 \\
Eccema & 4,566 & 0,033 & 0,62 \\
\hline
\end{tabular}

A cerca del consumo temprano de leche de vaca y su relación con el asma, los casos validos fueron 1322 siendo 187 los que contestaron haberla consumido antes de los seis meses y 1135 después de esta edad, se encontró que las variables son independientes con un OR: 1,2 una; $\mathrm{P}>0,05$, y un Chi-cuadrado de: 1,776 no relacionando el consumo de leche de vaca con el asma. Referente a la rinitis el total de casos fue de 1287 , siendo los que consumieron este alimento antes de los seis meses 178 y después de los seis meses 1 109, los resultados arrojaron un OR: 0,69 un Chi-cuadrado: 4,084 y una $\mathrm{P}<0,05$ hallándose relación entre el consumo temprano de leche y la rinitis alérgica como factor protector. En el caso del eccema y el consumo de leche el número de individuos válidos para el estudio ascendió a 1 260, siendo 178 los que la consumieron antes de los seis meses y 1082 quienes procuraron su ingesta después de los 6 meses, al analizar 
la información se obtuvo un Chi-cuadrado: O,142, un OR: 0,95 y una $\mathrm{P}>0,05$ se establece con un $95 \%$ de confianza que no hay relación entre el consumo de leche de vaca y la manifestación del eccema (Tabla 3).

Tabla 3. Presencia de asma, rinitis y eccema y consumo de leche de vaca

\begin{tabular}{lccc}
\hline Alergia & $\begin{array}{c}\text { Chi-cuadrado de } \\
\text { Pearson }\end{array}$ & $\begin{array}{c}\text { Significación asintótica } \\
\text { (bilateral) }\end{array}$ & $\begin{array}{c}\text { Odds ratio } \\
\text { (OR) }\end{array}$ \\
\hline Asma & 1,776 & 0,183 & 1,20 \\
Rinitis & 4,084 & 0,043 & 0,69 \\
Eccema & 0,142 & 0,706 & 0,95 \\
\hline
\end{tabular}

\section{DISCUSIÓN}

La introducción temprana de alimentos procesados a la dieta de los menores está comprobado provoca alteraciones en su sistema digestivo y en el sistema tegumentario (16) desfavoreciendo así la modulación y el buen desarrollo de su sistema inmune, también es posible que sea una de las causa principales en la aparición de alergias, dentro de los alimentos que presentan altos índices de provocar alergias encontramos al huevo, el pescado y la leche de vaca (7), evidenciándose que al momento de retirarlos de la dieta regular hay una tendencia a que mejore la condición de salud o en su defecto cesen por completo los episodios de alergias como es el caso del consumo de huevo (17), pero también se encuentran disponibles estudios que manifiestan que no existe total certeza que su consumo así sea a temprana edad sensibilice entorno a padecer alergias (18), al respecto a esto dentro de los resultados el estudio hallo similitud al anterior ya que por análisis estadístico de significancia no se estableció relación entre el consumo temprano de huevo y la presencia de asma rinitis y eccema.

Ante el consumo temprano de pescado y la presencia de enfermedades alérgicas, tampoco hay un consenso sobre si tomarlos o no, se observó que se refiere que su ingesta favorece el desencadenamiento de enfermedades alérgicas con episodios fuertes en los menores (19) como también se encuentra literatura que indica que su consumo incluso regular durante el año uno de vida y después de él pueden resultar favorecedor para que no se observen manifestaciones sintomáticas de alergias (20). En torno a esto, el consumo temprano de pescado refiere como resultado que su ingesta puede llegar a convertirse en un factor protector para el padecimiento de asma y eccema considerándose que esto se puede deber a que en el pescado hay ácidos grasos omega-3 ( $\omega-3)$ poliinsaturados que actuarían como desencadenantes para la inhibición de la respuesta alérgica (12) se presume que el resultado en el estudio se debe a que por factores culturales y de orden geográfico por encontrarnos en la región caribe hay un mayor acceso y disponibilidad en cuanto a la adquisición del alimento, mas no debemos desconocer que la base genética juega un papel fundamental en el desarrollo de enfermedades en especial las de índole alérgica (21) y no fue abordado el tópico si alguno de los padres había desarrollado o no las enfermedades.

En el consumo de leche de vaca en las primeras etapas de vida, se ha logrado establecer aunque de modo no conclusivo que su consumo temprano está asociado a episodios alérgicos, en gran medida debido a que el sistema digestivo aun inmaduro no logra procesarla de forma adecuada (22). Al respecto se puede establecer que por investigaciones que en muchos casos sobre alergias por leche de vaca solo se reportan las que se evidencian de forma casi que inmediata como los problemas gastrointestinales y las alergias cutáneas, no reportando las alergias como la rinitis (23) la investigación al respecto concuerda con lo expresado ya que no arrojo evidencia que ligara su consumo con síntomas de rinitis alérgica presentándola como factor protector.

A pesar que los resultados no son del todo concluyentes en lo que respecta a si el consumo de huevo, pescado y leche de vaca en edades tempranas se relacionan con enfermedades alérgicas cabe destacar que los individuos refirieron síntomas de estas inclusive si el alimento se introdujo después de los seis meses, ante este hecho se recomienda asumir la recomendación expresada por la oms en lo que respecta a procurar la introducción de alimentos después de los seis meses, y antes de ese tiempo brindar de forma exclusiva leche materna (24), esta investigación no tomó lo referente a lo genético y medio ambiental como factores predisponentes y desencadenantes de alergias (25), considerando que estos tópicos podrían ser tomados en cuenta como complemento de futuras investigaciones $\boldsymbol{e}$

\section{REFERENCIAS}

1. Smith $\mathrm{KR}$, Corvalan $\mathrm{CF}$, Kjellstrom T. How much global ill health is attributable to environmental factors? Epidemiology. 1999; 10(5):573-84.

2. Llamazares A. Epidemiología de las enfermedades alérgicas. Jano. Medicina y Humanidades. 2002; (63):44-47.

3. Kay AB. Allergy and Allergic Diseases. New England Journal Medicine. 2001; 344(1):30-37.

4. Gupta R, Sheikh A, Strachan DP, Anderson HR. Time trends in allergic disorders in the UK. Thorax. 2007; 62(1):91-6.

5. Sociedad Mexicana de Neumología y Cirugía de Tórax, A.C. Consenso Mexicano de Asma. Neumología y Cirugía de Tórax. 2005; 64(S1): S7- S44.

6. Pearce N, Aït-Khaled N, Beasley R, Mallol J, Keil U, Mitchell E, Robertson C. Worldwide trends in the prevalence of asthma symptoms: Phase III of the International Study of Asthma and Allergies in Childhood (ISAAC). Thorax. 2007; (62):757-765.

7. Kaplan A, Bousquet J, Naspitz C. Prevention of allergy and asthma. Allergy Clin. Immunology. Int. 2000; 12 (6):288-299. 
8. Romero-Tapia S. Prevención de alergia y Asma. Salud en Tabasco. 2002; 8(2):83-86.

9. Lack G. Clinical practice. Food allergy. New England Journal Medicine. 2008; 359(12):1252-60.

10. Medina-Ávalos M, Ruz-Cabrera C y Anaya-Cassou J. Conocimientos de familiares de pacientes alérgicos, población derechohabiente y personal de salud acerca de las enfermedades alérgicas. Revista de especialidades Médico-Quirúrgicas 2011; 16:13-17.

11. Rona RJ, Keil T, Summers C, Gislason D, Zuidmeer L, Sodergren E, McBride $D$. The prevalence of food allergy: A meta-analysis. Journal Allergy Clinical Immunology. 2007; 120(3):638-646.

12. Kull I, Wickman M, Lilja G, Nordvall SL, Pershagen G. Breastfeeding and allergic diseases in infants - a prospective birth cohort study. Archives of Disease Childhood. 2002; 87(6):478-81.

13. Tarini BA, Carroll AE, Sox CM, Christakis DA. Systematic review of the relationship between early introduction of solid foods to infants and the development of allergic disease. Archives of Pediatrics \& Adolescent Medicine 2006; 160(5):502-507.

14. Fallani M, Young D, Scott J, Norin E, Amarri S, Adan R, Dore J. Intestinal microbiota of 6-week-old infants across Europe: geographic influence beyond delivery mode, breastfeeding, and antibiotics. Journal pediatric gastroenterology and nutrition. 2010; 51(1):77-84.

15. Rosas-Vargas M A; González-Reyes M., Del Rio Navarro B E., Ávila Castañon L. Velázquez Armenta Y, Sienra Monje J. Sensibilización con alergenos asma en niños de 1 a 3 años de edad. Revista Alergia. 2002; 49(6):171-175.

16. Roy-Ghanta S, Larosa DF, Katzka DA. Atopic characteristics of adult patients with eosinophilic esophagitis. Clinical Gastroenterology and Hepatology. 2008; 6(5):531-535
17. Rojas R. Quezada A. Relación entre dermatitis atópica y alergia alimentaria. Revista Chilena de Pediatría. 2013; 84(4):438-450.

18. Sampson HA. Anaphylaxis and emergency treatment. Pediatrics. 2003; 111:1601-08.

19. Cattaneo A, Williams C, Pallás-Alonso CR, Hernández- Aguilar MT, Lasarte J, Landa-Rivera L, Oudesluys-Murphy A. ESPGHAN's 2008 recommendation for early introduction of complementary foods: how good is the evidence?. Maternal and Child Nutrition 2011; 7(4):335-43.

20. Morales-Romero C, Bedolla-Barajas M, López-Vargas L, Romero-Velarde C. Prevalencia de las enfermedades alérgicas y su asociación con la lactancia materna y el inicio de la alimentación complementaria en niños de edad escolar de Ciudad Guzmán, México. Archivos Argentinos de pediatría 2015; 113(4):324-330.

21. Sicherer SH, Sampson HA. Food allergy: Epidemiology, pathogenesis, diagnosis, and treatment. Journal of Allergy Clinical Immunology. 2014; 133(2):291-307.

22. Prescott SL. Early origins of allergic disease: a review of processes and influences during early immune development. Current opinion in allergy and clinical immunology. 2003; 3(2):125-132.

23. Miquel E Isabel, Arancibia S M. Eugenia. Alergia a proteína de leche de vaca en el menor de un año. Revista Chilena Pediatría. 2012; 83(1):78-83

24. Toca M, Tonietti M, Vecchiarelli C. Nutrición pre y posnatal: impacto a largo plazo en la salud. Archivos argentinos de pediatría. 2015; 113(3): 254-259.

25. Sánchez J, Restrepo M, Mopan J, Chinchilla C, Cardona R. Alergia a la leche y al huevo: diagnóstico, manejo e implicaciones en América Latina. Biomédica. 2014; 34(1):143-156. 